\title{
Information for Authors Submission Process
}

\section{Submission Process}

The manuscript submission process is broken into a series of 5 screens that gather detailed information about your manuscript and allow you to upload the pertinent files.

The sequence of screens are as follows:

1. A long form asking for author information, title, abstract, and file quantities.

2. A screen asking for the actual file locations on your computer (via an open file dialog). After completing this screen, your files will be uploaded to our server.

3. A screen requesting the order files should appear in the systemgenerated merged PDF.

4. A completion screen that will provide you with a specific manuscript number for your manuscript.

5. An approval screen that will allow you to verify that your manuscript was uploaded and converted correctly. You are allowed to replace and delete files, as well as withdraw the manuscript, on this page.

Before submitting a manuscript, please gather the following information:

- All Authors First Names, Middle Names/Initials, Last Names

- Author affiliations/Institutions

- Departments

- Phone and Fax Numbers

- Street Addresses

- E-mail Addresses

- Title and Running Title (you may copy and paste these from your manuscript) YOUR TITLE MUST BE UNDER 80 CHARACTERS (including spaces)

- Structured Abstract (unless a Review Article, then Unstructured)

\section{File Formats}

- Manuscript files in Word, WordPerfect, or Text formats

- Figures/Images in TIF, EPS, PDF, or JPG formats (must follow high resolution formats below)

- Tables in XLS or DOC formats

- Figure/File mode/Ideal resolution/Minimum resolution

- Line Bitmap 1200 dpi(ideal) 600 dpi(min)

- Color photo CMYK 300 dpi(ideal) 200 dpi(min)

- Black and White photos Grayscale 300 dpi(ideal) 200 dpi(min)

- Line/halftone combination Grayscale 600 dpi(ideal) 200 dpi(min)

Uniform Requirements for Manuscripts Submitted to Biomedical Journals: Writing and Editing for Biomedical Publication International Committee of Medical Journal Editors

For detailed instructions regarding style and layout refer to "Uniform requirements for manuscripts submitted to biomedical journals". Copies of this document may be obtained on the website http://www.icmje.org. Articles should be submitted under conventional headings of introduction, methods and materials, results, discussion, but other headings will be considered if more suitable. For Uniform Requirements for Sample References go to http://www.nlm.nih.gov/bsd/uniform_requirements.html.

After the manuscript is submitted, you will be asked to select the order you would like the files to be displayed in a merged PDF file that the system will create for you. Next, you will be directed to a page that will allow you to review your converted manuscript. If the conversion is not correct, you can replace or delete your manuscript files as necessary. You may also add additional files at this time. After you have reviewed the converted files, you will need to click on "Approve Converted Files." This link will have a red arrow next to it. Throughout the system, red arrows reflect pending action items that you should address.

\section{Cover Letter}

A cover letter is required and must state that the manuscript: has not been published elsewhere, except in abstract form, and is not under simultaneous consideration by another journal. Once a decision is made by the Editor on your manuscript, the Journal office will send you an Author Release form and a Conflict of Interest form if your manuscript has been accepted for revision.

\section{Abstracts}

Original Articles should be accompanied by a Structured abstract of 250 words or less on a separate page, in either English or French. The Journal will provide translation to the other language if required. Abstracts should consist of four paragraphs headed: Background (or objective), Methods, Results and Conclusions. Review articles should be accompanied by an Unstructured abstract of 150 words or less. Brief Communications (Case Reports) require no Abstract.

\section{Acknowledgements}

Acknowledgements, including recognition of financial support, should be typed on a separate page at the end of the text. The SI system (système international d'unités) should be used in reporting all laboratory data, even if originally reported in another system. Temperatures are reported in degrees celsius. English language text may use either British or American spelling, but should be consistent throughout.

\section{References}

References should be numbered in the order of their citation in the text. Those cited only in tables and legends for illustrations are numbered according to the sequence established by the first identification in the text of a particular table or illustration.

Titles of journals should be abbreviated according to the style used in Index Medicus. Cite references in numerical order according to their position in the Reference list in the text.

List all authors when there are six or fewer; for seven or more, list only the first three and add "et al".

For pagination (e.g., 33-7, not 33-37).

Provide the full title, year of publication, volume number and inclusive pagination for journal articles. Unpublished articles should be cited as [in press]. Do not reference unpublished or "submitted" papers; these can be mentioned in the body of the text.

Avoid "personal communications" and, if necessary, include them in the body of the text, not among the references. Reference citations should not include unpublished presentations or other nonaccessible material. Books or chapter references should also include the place of publication and the name of the publisher.

For Reference Guidelines go to: www.nlm.nih.gov/bsd/uniform_ requirements.html.

\section{Examples of correct forms of reference:}

Journals

1. Rose ME, Huerbin MB, Melick J, et al. Regulation of interstitial excitatory amino acid concentrations after cortical contusion injury. Brain Res. 2002; 935(1-2):40-6.

\section{Chapter in a book}

1. Meltzer PS, Kallioniemi A, Trent JM. Chromosome alterations in human solid tumors. In: Vogelstein B, Kinzler KW, editors. The genetic basis of human cancer. New York: McGraw-Hill; 2002. p. 93-113.

\section{Tables}

Type tables double-spaced on pages separate from the text. Provide a table number and title for each. Particular care should be taken in the preparation of tables to ensure that the data are presented clearly and concisely. Each column should have a short or abbreviated heading. Place explanatory matter in footnotes, not in the heading. Do not submit tables as photographs.

\section{Review Articles}

Review articles on selected topics are also published. They are usually invited, but unsolicited reviews will be considered. Review articles should be accompanied by an Unstructured abstract of 150 words or less. 


\section{Brief Communications}

Brief Communications (formerly Case Reports) are published on various topics and should be limited to approximately 9 doublespaced manuscript pages (3 Journal pages), including references (limit to approx. 5 references) and may include illustrations and tables. Brief Communications do not require an abstract.

\section{Editor Correspondence}

Correspondence to the Editor concerning matters arising in recent articles are welcome. Correspondence should be limited to two double-spaced pages and may include one illustration and a maximum of four references.

\section{Critically Appraised Topics (CATs)}

Current research in clinical neurosciences. Each CAT will appraise one or two recent research articles dealing with a particular topic. Meta-analyses and systematic reviews will also be considered if pertaining to evidence-based neurological/neurosurgical practice. A complete CAT is a one or 2 page summary that includes all of the following:

A brief title that summarizes the conclusion reached about the article.

Clinical Bottom Lines consisting of short statements summarizing the key "take-home" points. The clinical problem which cues the reader to the nature of the case. The clinical problem comes from real life dilemmas that are faced by clinicians. The clinical question includes the patient, intervention, comparator, and outcome.

The search strategy - including search terms, search engines used, and the reasons why the article chosen is the best evidence for the clinical question.

The evidence is described briefly including the type of study, patient population, and outcomes reported for the article reviewed.

The data is usually presented in tabular form and highlights the clinically significant data such as number needed to treat, specificity, hazard ratios, etc.

Comments are added regarding the quality of the study and any concerns which were identified by the critical appraisal process.

The reference, the appraiser, the date appraised, and the date expired.

Lastly, it will include a clinical comment from an "expert" on the particular topic.

\section{Neuroimaging Highlights}

Neuroimaging Highlights are selected by the Editor-in-Chief and Neuroimaging Highlight Editors on the basis of two factors. The first is high quality "state of the art" imaging of a novel and uncommon (or common with an uncommon twist) neurological or neurosurgical disorder. The second factor is the clinical novelty of the case.

Neuroimaging Highlights require a figure of several panels that clearly outlines all features of the relevant imaging. For example, for MR images this may require different cuts and sequences, etc. Combining more than one imaging modality strengthens the report. The report may also benefit from a single additional panel in a figure if it is directly relevant, e.g. a pathological image or patient image. The text should include a very brief discussion of the case history confined to the relevant history, pertinent abnormal findings, and clinical course with outcome. An additional one to two paragraphs should briefly describe the Neuroimaging panels present, and very briefly review relevant aspects of the literature. Overall, the Neuroimaging Highlights should be 500 words or less, with no more than 10 references.
Images should be of the highest quality, submitted electronically as a tif file at a minimum of $300 \mathrm{dpi}$ and at a size large enough for the printed journal (i.e. not less than 3 1/2" wide).

Suitability for publication is judged by a Neuroimaging Highlight Editor, the Editor-in-Chief and up to one additional external referee.

\section{Permissions and Releases}

Any non-original material (quotations, tables, figures) must be accompanied by written permission from the author and the copyright owner to reproduce the material in the Journal. Photographs of recognizable persons must be accompanied by a signed release from the legal guardian or patient authorizing publication.

\section{Conflict of Interest}

Authors who have non-scientific or non-academic gain, whether it be financial or other, from publishing their article are responsible for declaring it to the Editor. Any financial interest, research grant, material support, or consulting fee associated with the contents of the manuscript must be declared to the Editor. These guidelines apply to each author and their immediate families. Conflicts of interest are not necessarily wrong, nor do they necessarily change the scientific validity of research or opinion, but the Journal and readers should be aware of the conflict. If the Editor considers the conflict to compromise the validity of the paper, it will not be accepted for publication.

Authors, editorial staff and reviewers are asked to declare any relationship that would be considered as a conflict of interest whether or not they believe that a conflict actually exists. Information that the Journal receives about conflict or potential conflict will be kept confidential unless the Editor or Associate Editor considers it to be important to readers. Such conflicts will be published in the author credits or as a footnote to the paper, with knowledge of the authors.

\section{Getting Help}

If you need additional help, you can click on the help signs spread throughout the system. A help dialog will pop up with contextsensitive help.

\section{Manuscript Status}

After you approve your manuscript, you are finished with the submission process. You can access the status of your manuscript at any time via:

\section{Logging into the system with your password}

Clicking on the link represented by your manuscript tracking number and abbreviated title

Clicking on the "Check Status" link at the bottom of the displayed page

This procedure will display detailed tracking information about where your manuscript is in the submission/peer-review process.

\section{Starting}

The manuscript submission process starts by pressing the "Submit Manuscript" link on your "Home" page. Please make sure you have gathered all the required manuscript information listed above BEFORE starting the submission process. 

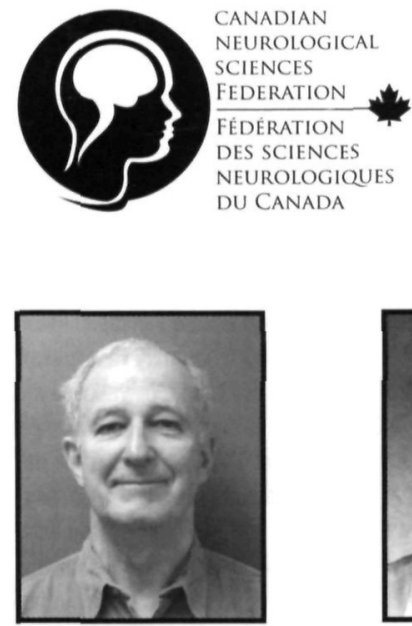

Derek Fewer

- CNSF President

- NSFC President

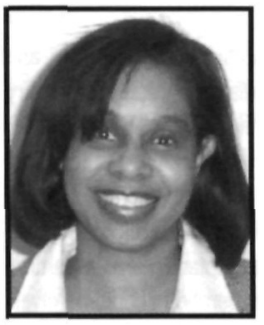

Sharon Whiting

- CNSF Board Member

- NSFC Board Member

- CACN Vice-President

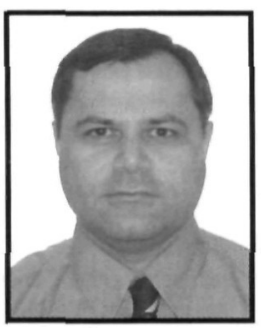

Seyed Mirsattari

- CNSF Board Member

- NSFC Board Member

- CSCN Vice-President

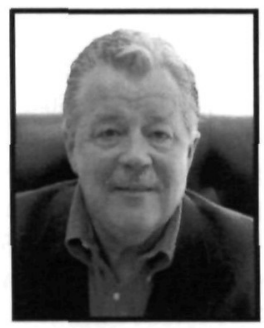

Dan Morin

- CEO

\section{The Canadian Neurological Sciences Federation (CNSF) and Neurological Sciences Foundation of Canada (NSFC)}

\section{0 - 2011 Board of Directors and Committee Chairs}

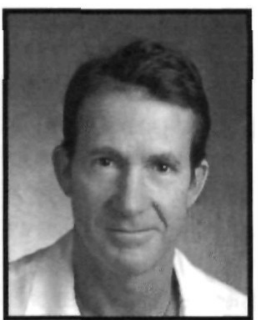

J. Max Findlay

- CNSF Vice-President

- NSFC Vice-President

- CNSS Past President

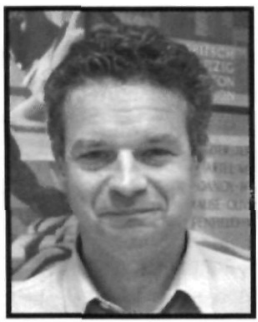

Chris Wallace

- CNSF Board Member

- NSFC Board Member

- CNSS President

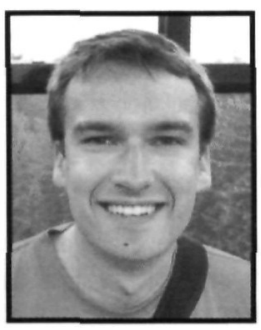

Trevor Steve

- CNSF Boara Member

- NSFC Board Member

- Residents' Rep. CNS

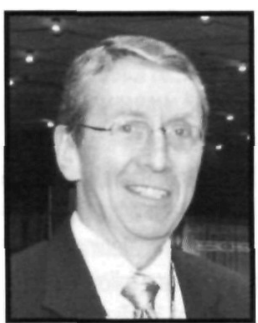

G. Bryan Young

- Journal Editor-in-Chief

- CNS \& CSCN Member

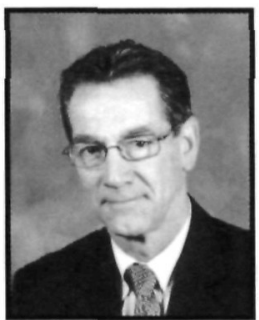

John Stewart

- CNSF Vice-President

- NSFC Vice-President

- CNS Member

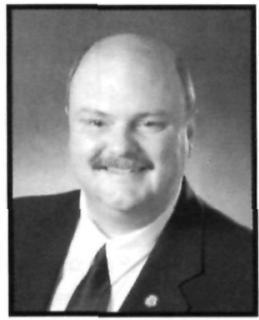

Lyle Weston

- CNSF Board Member

- NSFC Board Member

- CNS President

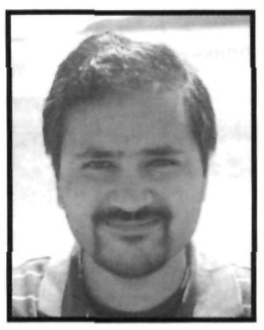

Shobhan Vachhrajani

- Residents' Rep. CNSS

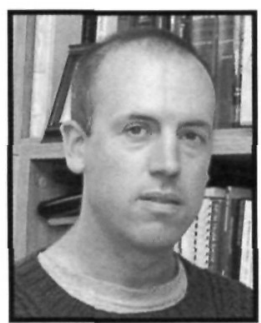

Michael Hill

- CNSF Scientific Program

Committee Chair

- CNS Member

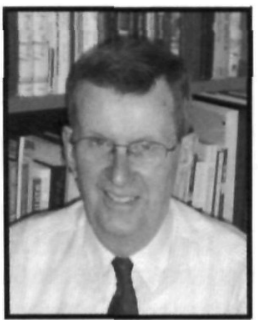

Garth Bray

- CNSF Executive VP

- NSFC Executive VP

- CNS Member

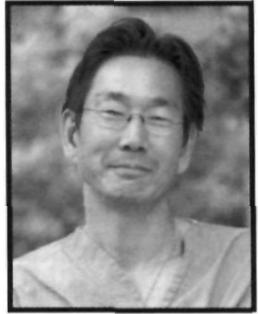

Brian Toyota

- CNSF Board Member

- NSFC Board Member

- CNSS Vice-President

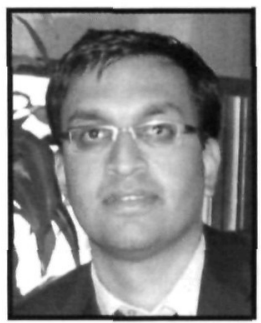

Vijay Ramaswamy

- Residents' Rep. CACN

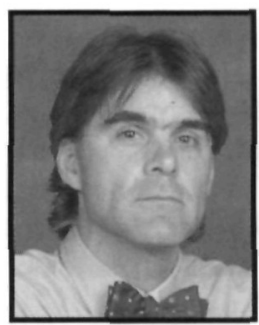

Colin Chalk

- CNSF PDC Chair

- CNS Member

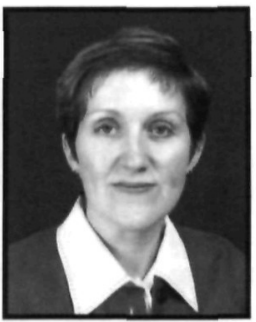

Mary Connolly

- CNSF Board Member - NSFC Board Member - CACN President

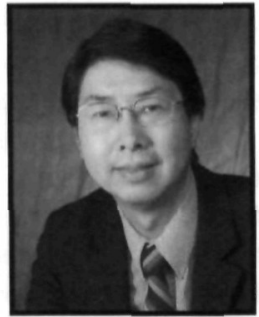

Ming Chan

- CNSF Board Member

- NSFC Board Member - CSCN President

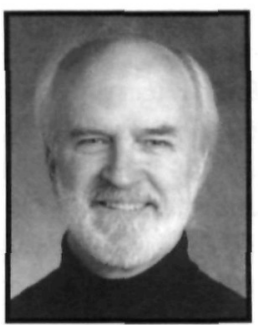

George Elleker

- CNSF/NSFC

Past President

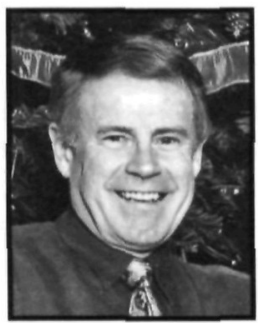

Richard Riopelle

- CBANHC Chair

Sarah Kirby CNSF/NSFC Board Member, CNS Vice-President Photo Unavailable

\section{Legend:}

CNSF - Canadian Neurological Sciences Federation; NSFC - Neurological Sciences Foundation of Canada; CNS - Canadian Neurological Society;

CNSS - Canadian Neurosurgical Society; CSCN - Canadian Society of Clinical Neurophysiologists; CACN - Canadian Association of Child Neurology;

CBANHC - Canadian Brain and Nerve Health Coalition 


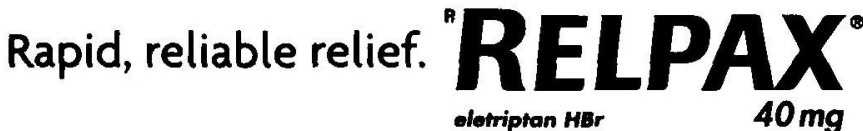

\section{Prescribing Summary}

\section{(I) Patient Selection Criteria}

THERAPEUTIC CLASSIFICATION: Migraine Therapy

INDICATIONS AND CLINICAL USE

RELPAX (eletriptan hydrobromide) is indicated for the acute treatment of migraine with or without aura in adults.

RELPAX tablets are not intended for the prophylactic therapy of migraine or for use in the management of hemiplegic, ophthalmoplegic or basilar migraine (See CONTRAINOICATONS). Safety and effectiveness of RELPAX tablets have not been established for cluster headache, which is present in an older, predominantly male population

\section{CONTRAINDICATIONS}

RELPAX tablets are contraindicated in patients with history, symptoms, or signs of ischemic cardiac, cerebrovascular or peripheral vascular syndromes, valvular heart disease or cardiac arthythmias (especially tachycardias). In addition, patients with other significant underlying cardiovascular diseases (e.g., atherosclerotic disease, congenital heart disease) should not receive eletriptan. Ischemic carciac syndromes include, but are not restricted to, angina pectoris of any type (e.g., stable angina of effort and vasospastic forms of angina such as the Prinzmetal's variant), all forms of myocardial infarction, and silent myocardial ischemia. Cerebrovascular syndromes include, but are not limited to, strokes of any type as well as transient ischemic attacks (TIAs). Peripheral vascular disease includes, but is not limited to, ischemic bowel disease, or haynaud's syndrome (see WARNINGS AND PRECAUTIONS).

Because RELPAX may increase blood pressure, it is contraindicated in patients with uncontrolled or severe hypertension (see WARNINGS AND PRECAUTIONS).

Eletriptan is metabolized by the CYP3A4 enzyme. Therefore, RELPAX is contraindicated within $72 \mathrm{~h}$ of treatment with the following potent CYP3A4 inhibitors: ketoconazole, itraconazole, nefazodone, troleandomycin, clarithromycin, ritonavir, and nelfinavir. RELPAX is contraindicated within $72 \mathrm{~h}$ with drugs that have demonstrated potent CYP3A4 inhibition and have this potent effect described in the CONTRAINDICATIONS, or WARNINGS AND PRECAUTIONS sections of their labeling (see DRUG INTERACTIONS and ADMINISTRATION).

RELPAX is contraindicated within $24 \mathrm{~h}$ of treatment with another $5-\mathrm{HT}_{\text {, agonist, }}$ an ergotamine-containing or ergot-type medication such as dihydroergotamine (DHE) or methysergide.

RELPAX is also contraindicated in patients with hemiplegic, ophthalmoplegic or basilar migraine; in patients with severe hepatic impairment; and in patients with known hypersensitivity to eletriptan or any of its inactive ingredients.

\section{SPECIAL POPULATIONS}

Pregnant women

The safety of eletriptan in pregnant women has not been established. Administration of RELPAX tablets should only be considered if the expected benefit to the mother is greater than any possible risk to the fetus (see Supplemental Product Information).

\section{Nursing women}

Caution should be exercised when RELPAX tablets are administered to nursing women Eletriptan is excreted in human breast milk (see Supplemental Product Information)

Pediatrics ( $<18$ years of age)

Safety and effectiveness of RELPAX tablets in pediatric patients have not been established; therefore, RELPAX is not recommended for use in patients under 18 years of age.

The efficacy of RELPAX tablets ( $40 \mathrm{mg}$ ) in patients 11-17 was not established in a randomized, placebo-controlled trial of 274 adolescent migraineurs.

Geriatrics ( $>65$ years of age)

RELPAX has been given to only 50 patients over the age of 65 . Blood pressure was increased to a greater extent in elderly subjects than in young subjects. Experience of the use of RELPAX in patients aged $>65$ years is limited. Therefore, the use of RELPAX in patients over 65 years is not recommended (see Supplemental Product Information).

\section{Safety Information}

\section{WARNINGS AND PRECAUTIONS}

\section{General}

RELPAX tablets should only be used where a clear diagnosis of migraine has been established.

\section{CYP3A4 inhibitors}

SEe CONTRANDICATIONS above.

Cardiovascular

Risk of myocardial ischemia and/or infarction and other cardiac events: As with other triptans, eletriptan has been associated with transient pain or pressure sensation in the chest or throat. Because of the potential of 5-HT, agonists to cause coronary vasospasm, eletriptan should not be given to patients with documented ischemic or vasospastic coronary artery disease (CAD) (see CONTRAINDICATIONS). It is strongly recommended that eletriptan not be given to patients in whom unrecognized CAD is predicted by the presence of risk factors (e.g., hypertension, hypercholesterolemia, smoker, obesity, diabetes, strong family history of $\mathrm{CAD}$, female with surgical or physiological menopause, or male $>\mathbf{4 0}$ years of age) unless a cardiovascular evaluation provides satisfactory clinical evidence that the patient is reasonably free of coronary artery and ischemic myocardial disease, or other significant underlying cardiovascular disease. The sensitivity of cardiac diagnostic procedures to detect cardiovascular disease or predisposition to coronary artery vasospasm is modest, at best. If, during the cardiovascular evaluation, the patient's medical history, electrocardiographic, or other investigations reveal findings indicative of, or consistent with, coronary artery vasospasm or myocardial ischemia, eletriptan should not be administered (see CONTRANDICATIONS).

These evaluations, however, may not identify every patient who has cardiac disease, and in very rare cases, serious cardiae events, such as myocardial infarction or coronary ischemia have occurred in patients without evidence of undertying cardiovascular disease. For patients with risk factors predictive of $C A D$ who are determined to have a satisfactory cardiovascular evaluation, it is strongly recommended that administration of the $1^{\text {st }}$ dose of eletriptan take place in the setting of a physician's office or similar medically staffed and equipped facility unless the patient has previously received eletriptan. Because cardiac ischemia can occur in the absence of clinical symptoms, consideration should be given to obtaining, on the $1^{\text {st }}$ occasion of use, an electrocardiogram (ECG) during the interval immediately following administration of eletriptan, in patients with risk factors. However, an absence of drug-induced cardiovascular effects on the occasion of the initial dose does not preclude the possibility of such effects occurring with subsequent administrations. It is recommended that patients who are intermittent long-term users of 5-HT, agonists including eletriptan, and who have or acquire risk factors predictive of CAD, as described above, undergo periodic cardiovascular evaluation as they continue to use eletriptan. If symptoms consistent with angina occur after the use of eletriptan, ECG evaluation should be carried out to look for ischemic changes.

The systematic approach described above is intended to reduce the likelihood that patients with unrecognized cardiovascular disease will be inadvertently exposed to therapy with eletriptan.

Discomfort in the chest, neck, throat and jaw (including pain, pressure, heaviness, and tightness) has been reported after administration of eletriptan. Because 5 -HT, agonists may cause coronary vasospasm, patients who experience signs or symptoms suggestive of angina following eletriptan should be evaluated for the presence of CAD or a predisposition to variant angina before receiving additional doses, and should be monitored electrocardiographically if dosing is resumed and similar symptoms recur. Similarly, patients who experience other symptoms or signs suggestive of decreased arterial flow, such as ischemic bowel syndrome or Raynaud's syndrome following eletriptan should be evaluated for atherosclerosis or predisposition to vasospasm (see CONTRAINDICATIONS and WARNINGS AND PRECAUTIONS and ADVERSE REACTIONS, Clinical trial adverse drug reactions).

Cardiac events and fatalities associated with 5-HT, agonists: As with other triptans, eletriptan may cause coronary artery vasospasm. Serious adverse cardiac events, including acute myocardial infarction, life-threatening disturbances of cardiac rhythm, and death have been reported within a few hours following the administration of other $5-\mathrm{HT}_{1}$ agonists. Considering the extent of use of $5-\mathrm{HT}$, agonists in patients with migraine, the incidence of these events is extremely low.

Patients with symptomatic Wolff-Parkinson-White syndrome or arrhythmias associated with other cardiac accessory conduction pathway disorders should not receive RELPAX.

As with other $5-\mathrm{HT}$, agonists, sensations of tightness, pain, pressure, and heaviness have been reported after treatment with RELPAX tablets in the precordium, throat and jaw. Events that are localized to the chest, throat, neck and jaw have not been associated with arrhythmias or ischemic ECG changes in clinical trials.

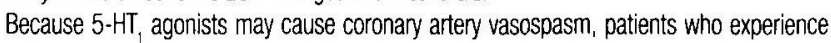
signs or symptoms suggestive of angina following dosing should be evaluated for the presence of $C A D$ or a predisposition to Prinzmetal's variant angina before receiving 
additional doses of medication, and should be monitored electrocardiographically if dosing is resumed and similar symptoms recur. Similarly, patients who experience other symptoms or signs suggestive of decreased arterial flow, such as ischemic bowel syndrome or Raynaud's syndrome, following the use of any $5-\mathrm{HT}$, agonist are candidates for further evaluation (see CONTRAINDICATIONS and Supplemental Product Information). Cerebrovascular events and fatalities associated with 5-HT, agonists: Cerebral hemorrhage, subarachnoid hemorrhage, stroke, and other cerebrovascular events have been reported in patients treated with 5.HT, agonists, and some have resulted in fatalities. In a number of cases, it appears possible that the cerebrovascular events were primany, the agonist having been administered in the incorrect belief that the symptoms experienced were a consequence of migraine, when they were not. It should be noted that patients with migraine may be at increased risk of certain cerebrovascular events (e.g., stroke, hemorrhage, transient ischemic attack).

Increase in blood pressure: Significant elevation in blood pressure, including hypertensive crisis, has been reported on rare occasions in patients receiving other $5-\mathrm{HT}$, agonists with and without a history of hypertension. In clinical pharmacology studies, oral eletriptan (at doses of $60 \mathrm{mg}$ or more) was shown to cause small transient dose -related increases in blood pressure, predominantly diastolic, consistent with its mechanism of action and with other 5- $\mathrm{HT}_{1 \mathrm{Ru}}$ agonists. The effect was more pronounced in renally impaired and elderly subjects. A single patient with hepatic cirrhosis received eletriptan $80 \mathrm{mg}$ and experienced a blood pressure of $220 / 96 \mathrm{mmHg} 5 \mathrm{~h}$ alter dosing. The treatment-related event persisted for $7 \mathrm{~h}$.

RELPAX tablets are contraindicated in patients with uncontrolled or severe hypertension (SEe CONTRAINDICATIONS)

Hepatic

The effects of severe hepatic impairment on eletriptan metabolism were not evaluated. RELPAX tablets should not be given to palients with severe hepatic impairment.

No dose adjustment is necessary in mild to moderate impairment (see ADMINISTRATION and Supplemental Product Information).

Neurologic

Care should be taken 10 exclude other potentially serious neurologic conditions before treating headache in patients not previously diagnosed with migraine headache or who experience a headache that is atypical for them. There have been rare reports where patients received 5-HT, agonists for severe headaches that were subsequently shown to have been secondary to an evolving neurologic lesion. For newly diagnosed patients or patients presenting with atypical symptorns, the diagnosis of migraine should be recorsidiered if IK) response is seen after the $1^{\mathrm{s}}$ dose of eletriptan.

Seizures: Caution should be observed if eletriptan is to be used in patients with a history of seizures or other risk factors, such as structural brain lesions, which lower the convulsion threshold.

Psychomotor effect

Patients should be advised to awoid driving a car or operating hazardous machinery until they are reasonably certain that RELPAX does not affect them adversely.

Renal

There was no significant change in clearance observed in subjects with mild, moderate or severe renal impairment. In some of these patients, an elevation in blood pressure was observed (see ADMINISTRATION).

Sensitivity/resistance

Hypersensitivity: Owing to the possibility of cross-reactive hypersensitivity reactions, RELPAX should not be used in patients having a history of hypersensitivity to chemically-related 5-HT, receptor agonists (see ADVERSE REACTIONS and Supplemental Product Information).

\section{ADVERSE REACTIONS}

Adverse drug reaction overview

Serious cardiac events, including some that have been fatal, have occurred following the use of other $5-\mathrm{HT}_{1}$ agonists. These events are extremely rare and most have been reported in patients with risk factors of CAD. Events reported have included coronary artery vasospasm, transient myocardial ischemia, myocardial infarction, ventricular tachycardia, and ventricular fibrillation (see CONTRAINDICATIONS and WARNINGS AND PRECAUTIONS).

Typical 5- $\mathrm{HT}_{1}$ agonist adverse reactions

As with other 5-HT, agonists, RELPAX has been associated with sensations of heaviness, pressure, tightness or pain which may be intense. These may occur in any part of the body including the chest, throat, neck, jaw and upper limbs.

Increases in blood pressure

Significant elevations in systemic blond pressure, including hypertensive crisis, have been reported on rare occasions in patients with and without a histony of hypertension treated with other 5 -HT, agonists. REL.PAX is contraindicated in patients with uncontrolled hypertension (SEe CONTRAINDICATIONS).

Clinical trial adverse drug reactions

Among 5,984 patients who treated a single migraine headache with RELPAX 20,40 or $80 \mathrm{mg}$ tablets in short-term, placebo controlled trials, the most common and dose-related adverse events (AEs) reported with treatment with REL PAX were asthenia $(7.2 \%)$, nausea $(7.8 \%)$, diziness $(5.7 \%)$ and somnolence (5.2\%) (see Supplemental Product Information and Table 1 below). RELPAX tablets are generally well tolerated. Across all doses, most AEs were mild and transient. The frequency of AEs in clinical trials did not increase when up to 2 doses of RELPAX tablets were taken within $24 \mathrm{~h}$. The incidence of AEs in controlled clinical trials was not affected by gender, age, or race of patients. AE frequencies were also unchanged by concomitant use of drugs commoniy taken for migraine prophylaxis, (e.g., SSRis, beta-blockers, calcium channel blockers, tricyclic antidepressants), estrogen replacement therapy and oral contraceptives.

\section{DRUG INTERACTIONS}

Effects of other drugs on eletriptan

CYP3A4 inhibitors: See CONTRAINDICATIONS and Supplemental Product Information.

Ergot-containing drugs: Ergot-containing drugs have been reported to cause prolonged vasospastic reactions. Because these effects may be additive, use of ergotamine-containing or ergot-type medications (like dihydroergotamine [DHE] or methysergide) and RELPAX tablets within $24 \mathrm{~h}$ is not recommended (see CONTRAINDICATIONS).

Other 5-HT, agonists: See CONTRAINDICATIONS.

Selective serotonin reuptake inhibitors (SSRIs): SSRIs (e.g., fluoxetine, fluvoxamine, paroxetine, sertraline) have been reported, rarely, to cause weakness, hyperreflexia, and incoordination when co administered with $5 \cdot \mathrm{HT}_{1}$ agonists. If concomitant treatment with eletriptan and an SSRl is clinically warranted, appropriate observation of the patient is advised. Drug-food interactions

The AUC and $\mathrm{C}_{\mathrm{Tar}}$ of eletriptan are increased by approximately 20 to $30 \%$ following oral administration with a high fat meal.

Health Canada may be notified by phone of serious or unexpected reaction to this drug at: $1.866 \cdot 234-2345$.

\section{$\Delta \theta$ Administration}

Dosing considerations

RELPAX tablets should be taken as early as possible after the onset of a migraine attack, but are also effective if taken at a later stage. RELPAX tablets should not be used prophylactically. Recommended dose and dosage adjustment

Adult (18-65 years of age): in controlled clinical trials, single doses of $20 \mathrm{mg}$ and $40 \mathrm{mg}$ were effective ior the acute trealment of migraine in adults. A greater proportion of patients had a response following a $40 \mathrm{mg}$ dose than following a $20 \mathrm{mg}$ dose. Individuals may vary in response to doses of RELPAX tablets.

When initiating treatment with RELPAX, a starting dose of $20 \mathrm{mg}$ or $40 \mathrm{mg}$ may be considered. Patients who do not obtain salisfactory efficacy after an intial trial of $20 \mathrm{mg}$ may be effectively treated with $40 \mathrm{mg}$ in subsequent migraine attacks. The choice of dose should therefore be made on an individual basis, according to the clinical status of the patient and weighing the possible riskfbenefit of the $40 \mathrm{mg}$ dose. A minimal effective dose should be used.

If after an initial dose of $20 \mathrm{mg}$, headache improves but then returns, a repeat dose of $20 \mathrm{mg}$ may be beneficial and should be taken at least $2 \mathrm{~h}$ after the initial dose. If an initial dose of $40 \mathrm{mg}$ is taken, a $2^{\text {nut }}$ dose is not recommended.

If the initial dose is ineffective, controlled clinical trials have not shown a benefit of a $2^{n}$ dose to treat the same attack.

The maximum daily dose should not exceed $40 \mathrm{mg}$.

The safety of treating an average of more than 3 headaches in a 30-day period has not been established.

Patients receiving potent CYP3A4 inhibitors

Eletriptan is metabolized by the CYP3A4 enzyme. Concomitant use of RELPAX and potent CYP3A4 inhibitors may lead to significant increases in AUC and $C_{\text {nar }}$, therefore RELPAX tablets are contraindicated within $72 \mathrm{~h}$ of treatment with the following potent CYP3A4 inhibitors: ketoconazole, itraconazole, clarithromycin, troleandomycin, ritonavir, nelfinavir and nefarodone. RELPAX is contraindicated within $72 n$ with drugs that have demonstrated potent CYP3A4 inhibition and have this potent effect described in the CONTRAINDICATIONS or WARNINGS AND PRECAUTIONS sections of their labeling (see DRUG INTERACTIONS and CONTRAINDICATIONS).

\section{Patients with hepatic impairment}

No dose adjustment is required in patients with mild or moderate hepatic impairment. As RELPAX has not been studied in patients with severe hepatic impairment, it is contraindicated in these patients (see CONTRAINDICATIONS).

Patients with renal impairment

In some patients with renal impairment, an elevation in blood pressure was observed. A total daily dose of greater than $20 \mathrm{mg}$ should be administered with caution (see WARNINGS ANO PRECAUTIONS).

\section{Administration}

RELPAX tablets should be swallowed whole with water. 


\section{Study References}

1. RELPAX Product Monograph, Pfizer Canada Inc., March 2006.

2. Sheftell $F$ et al. Efficacy, safely, and tolerability of oral eletriptan for treatment of acute migraine: a multicenter, double-blind, placebo-controlled study conducted in the United States. Headache 2003:43:202-213.

3. Mathew NT et al. Comparative efficacy of eletriptan $40 \mathrm{mg}$ versus sumatriptan $100 \mathrm{mg}$. Headache 2003:43:214-222

4. Sandrini $G$ et al: Eletriptan vs sumatriptan: a double-blind, placebo-controlled, multiple migraine attack study. Neurology 2002;59:1210-1217.

\section{Supplemental Product Information}

WARRIIINGS ARO PRECAUTIONS

Pregnant women

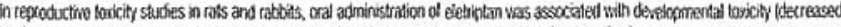

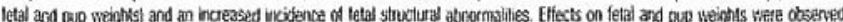

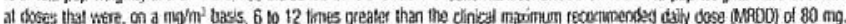

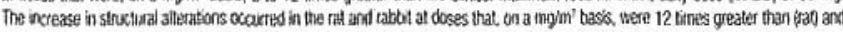

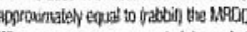

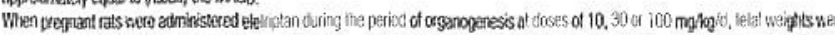

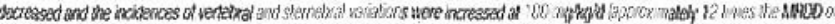

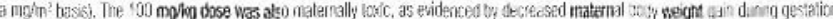

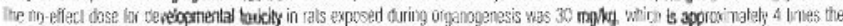
WPOD m a mghro basis.

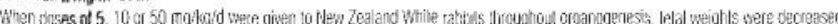

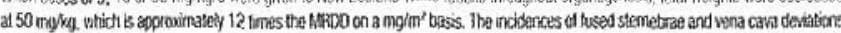

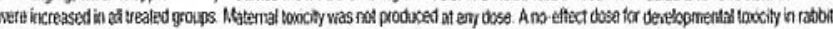

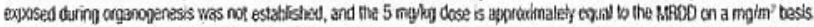

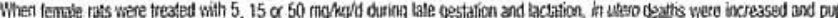

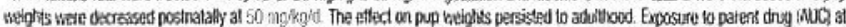

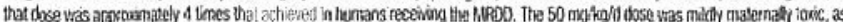

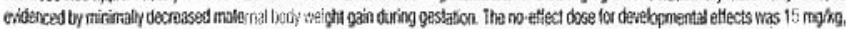

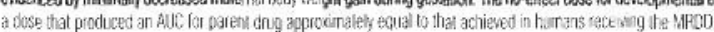

\section{Nursing women}

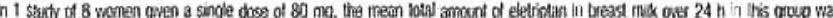
aprow

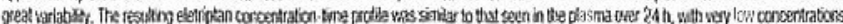

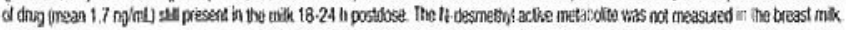
Geriatrics ( $>65$ years ol age)

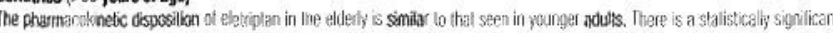

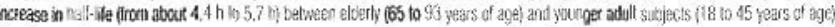
Cardiouascular

\section{Cardikg events and fatalities assoctated with 5-HT, agonists:}

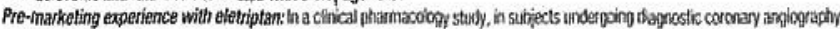

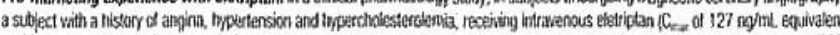

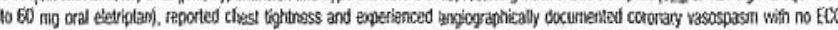

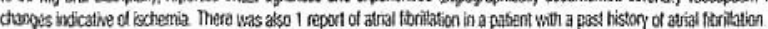

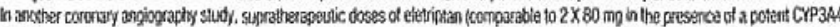

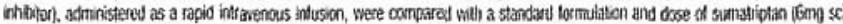

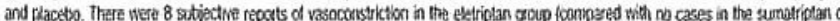

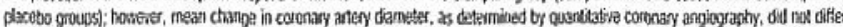
in the 3 treatrinent croups

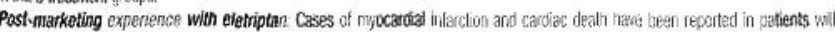

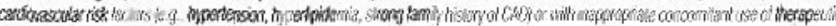

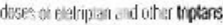

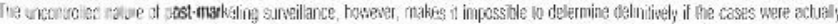

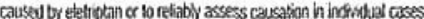

Special cardiovascular pharmacology studies with another $5 \cdot \mathrm{HT}$, agonist: In sutjects (n=10) wath suspocted CAs) undergcing

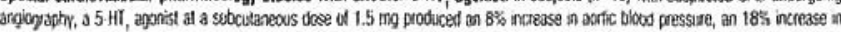

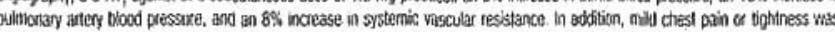

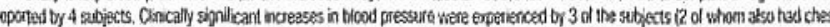

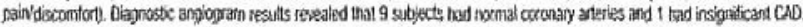

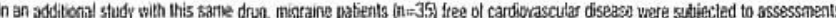

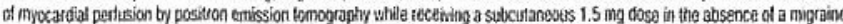

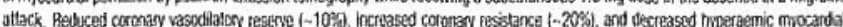

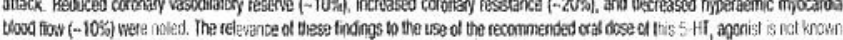

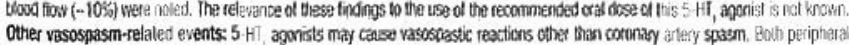

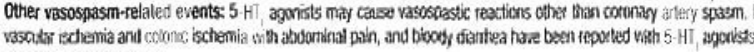
Dependenceit tolerance

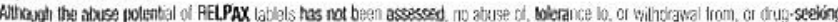

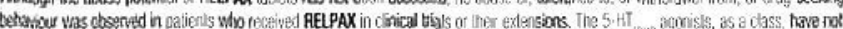
been asscciared with ditur atuse

Hepatic

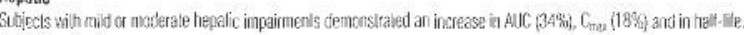
Ophthalmologic

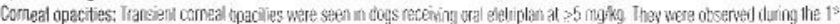

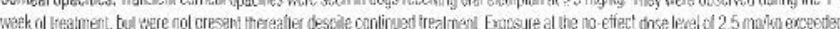
Inal acheved in hiurans at Ria Wido.

Preclinical toxicology

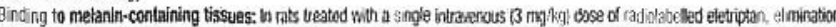

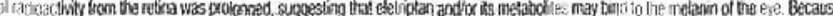

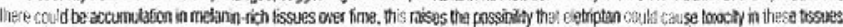

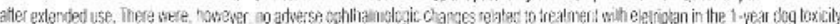

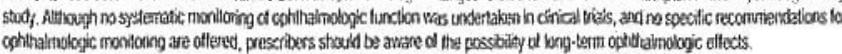

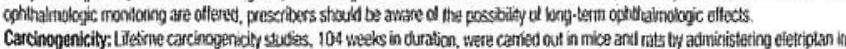

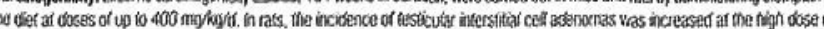

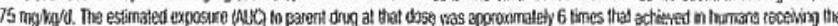

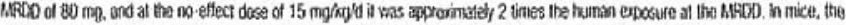

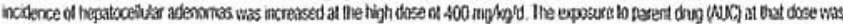

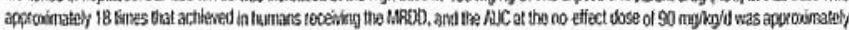

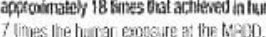

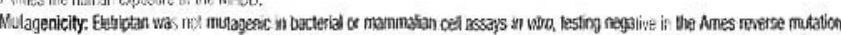

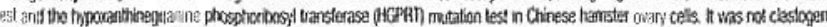

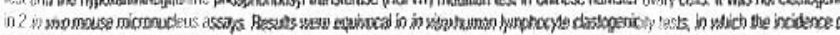

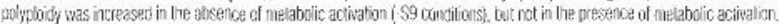

Sensitivityiresistance

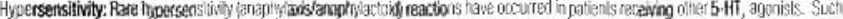

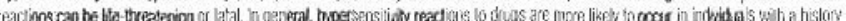
of sensiltyty to mullipe allacens scre ADVCRSE PEACTIONS)

\section{Sexual function/reproduction}

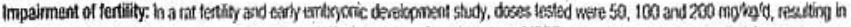

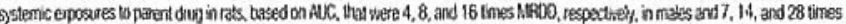

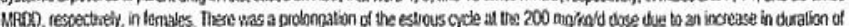

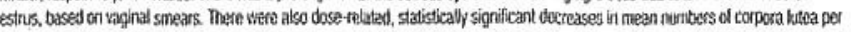

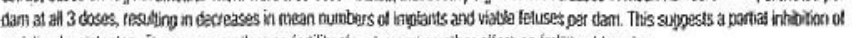

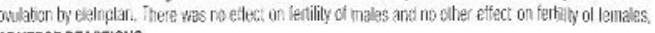

ADVERSE AEACTIONS

Clinical trial adverse drug reactions

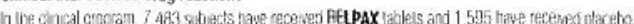

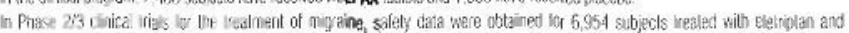

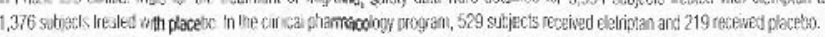

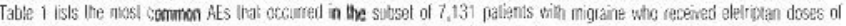

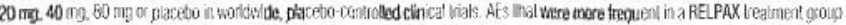

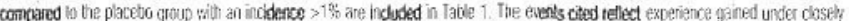

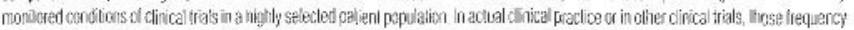

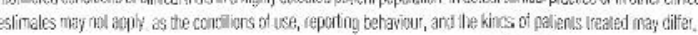

Table 1. Treatment-emergent adverse events by inilial oral dose of RELPAX and placebo reporled by $\geq 1 \%$ patients with migraine trom controlled ellinical trials

\begin{tabular}{|c|c|c|c|c|}
\hline & Placebo & $20 \mathrm{mg}$ & $40 \mathrm{mg}$ & $80 \mathrm{mg}$ \\
\hline Number of patients & 1559 & 536 & 2951 & 2085 \\
\hline \multicolumn{5}{|l|}{ Symptoms of potentially cardiac origin } \\
\hline Chesi sencation: & 1.1 & 0.4 & 22 & 4,4 \\
\hline Neckithoaliaw sensations" & 0.2 & 0.2 & 1.4 & 2.2 \\
\hline Palpitations & 0.9 & 0.7 & 1.3 & 1.8 \\
\hline Upoer Imb sensations* & 0.1 & 0.2 & 06 & 1.1 \\
\hline \multicolumn{5}{|l|}{ Neurological } \\
\hline Dominess & 2.8 & 2.4 & 5.1 & 7.2 \\
\hline Dronsiness & 28 & 1.9 & 4,9 & 59 \\
\hline Hetwlace sensalions. & 07 & 1.5 & 1.2 & 1.8 \\
\hline Heathche & 2.4 & 28 & 28 & 35 \\
\hline Hepertorib & 0.2 & 0.9 & 0.6 & 1.8 \\
\hline vertigo & 0.5 & 02 & 04 & 18 \\
\hline \multicolumn{5}{|l|}{ Digestive } \\
\hline Abdominal discomfort \& $p 3$ in & 0.7 & 09 & 1.7 & 22 \\
\hline Diaritez & 0.9 & 1,1 & 1.1 & 14 \\
\hline Gasirointestinal discomlent \& pain & 0.8 & 1.9 & 16 & 2.3 \\
\hline Hyposalvation & 1.5 & 2,1 & 30 & 3.7 \\
\hline Nausea & 7,8 & 3.9 & 6.9 & 10.4 \\
\hline Voniting & 5.7 & 0.6 & 30 & 4.0 \\
\hline \multicolumn{5}{|l|}{ Musculoskeletal } \\
\hline Muscle alophy, veakriess \& tiredness & 0.5 & 0.2 & 08 & 30 \\
\hline Muscle gain & 0.4 & 1.1 & 1.5 & 29 \\
\hline \multicolumn{5}{|l|}{ Ear, nose \& throat } \\
\hline Nasal sgns \& symplens & 0.6 & 0.9 & 10 & 15 \\
\hline Throas \& tensil symptoms & 0.4 & 1.3 & 1.4 & 24 \\
\hline \multicolumn{5}{|l|}{ Respiratory } \\
\hline Wral iniection & 0.8 & 0.6 & 1.1 & 1.3 \\
\hline \multicolumn{5}{|l|}{ Non-site specific } \\
\hline Chils & 1.3 & 0.2 & 0.8 & 1.2 \\
\hline Malaisellatigue & 1.9 & 26 & 4.5 & 94 \\
\hline Sensations & 2.1 & 2.6 & 36 & 56 \\
\hline Swealing & 0.6 & 0.4 & 1,1 & 1.6 \\
\hline
\end{tabular}

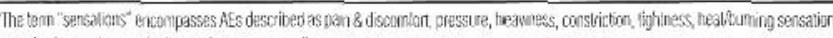
paresthesia, numbness, tingling and strange sensaligns.

Other events observed in association with the administration of PELPAX lablets

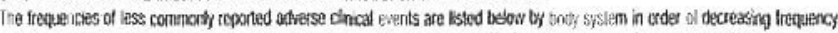

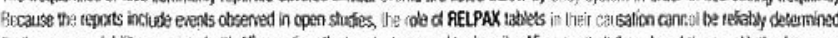

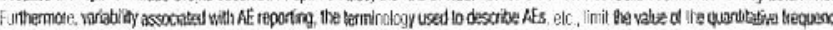

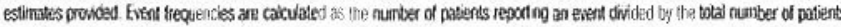

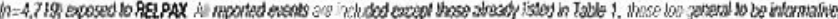

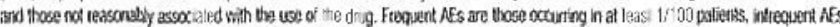

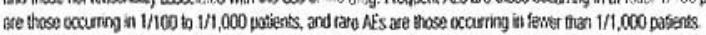

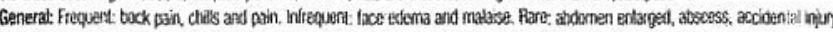

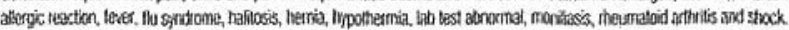

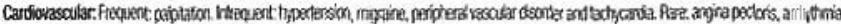

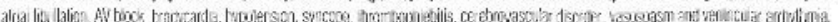

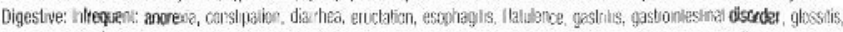

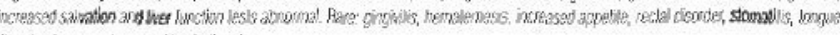
dsurder, longue exema and tonth disooder.

Endocrine: Fise goiler, llyrod aderoma and ligrodiditis,

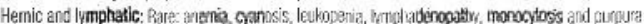

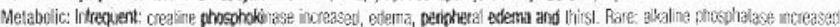

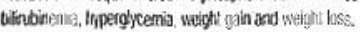

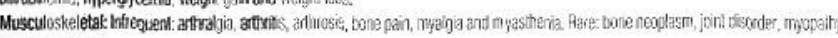
and lencegnowitis.

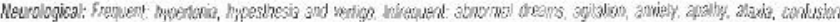

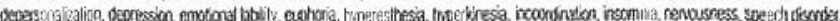

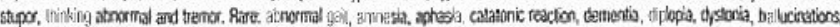

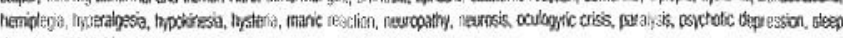
disorder and hacctings

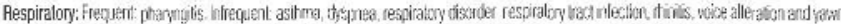

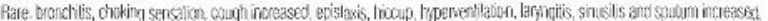

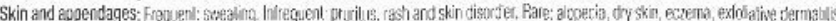

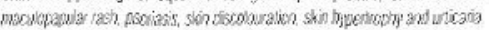

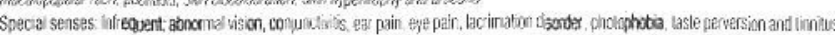

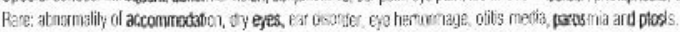

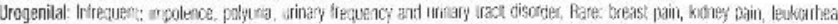
mencrithaia, mengthus disoder and vaginitis.

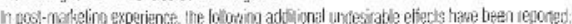

Gasto-intestinal disorders: ischaenc, oulis:

Nervous systenu disorders: Sproget:

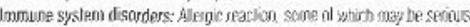

Skin and subrataneous tissue disorders: Prinhus, rash, uticana. 


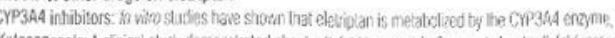

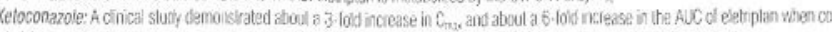

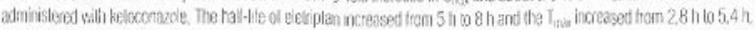

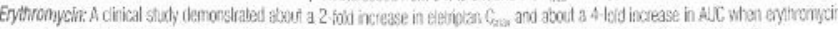

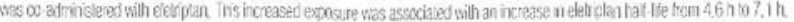

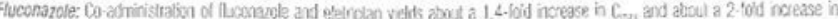
mucol eteriplan

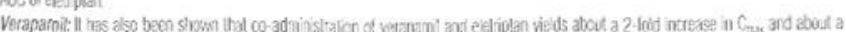
3. lots increase in AuC ol elevintan

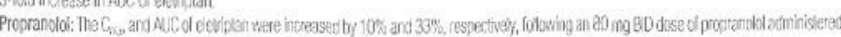

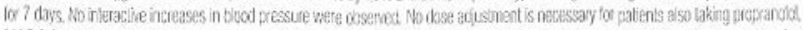

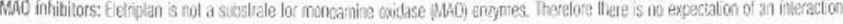

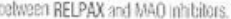

The effect of e'etriptan on other drugs

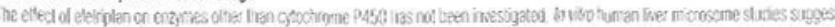

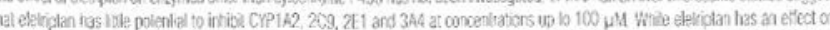

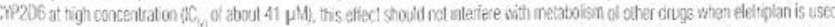

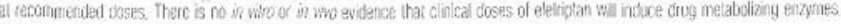

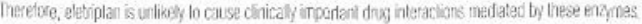

Orug-herb interactions

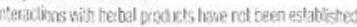

Orug-laboratory interactions

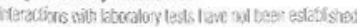

SYMPTOWS AND TREATMENT OF OVERDOSE

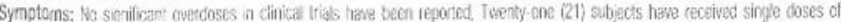

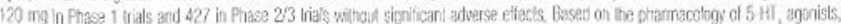

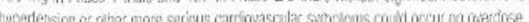

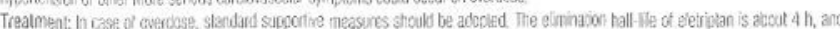

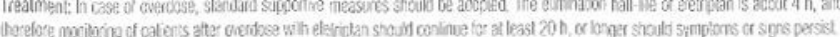

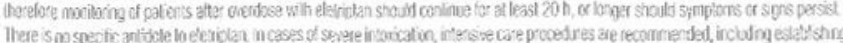

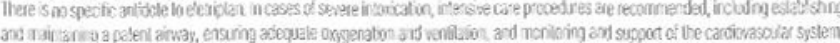

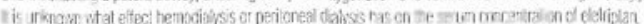

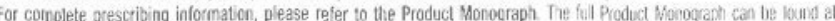

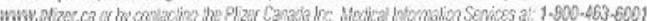

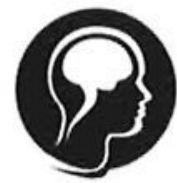

\section{ADVERTISERS INDEX}

Athena Diagnostics

CNSF Sponsors

Elekta

Interior Health

King Medical

Pfizer

$$
\text { -Relpax }
$$

$\mathrm{OBC}$

A-6

IFC

Scotia Bank

A-16

A-15

Teva Canada Inovation

Vancouver Island

Health Authority
A-3

A-7, A-12 to A-15

IBC, A-8

A-15
KING MEDICAL THE CANADIAN ELECTRODE PLACE

- ALPINE BIOMED Mono/Conc. Needles

- AMBU Blue Sensor - Neuroline

- CHALGREN Needles - Bar/Ring/Clip

- KENDALL Adhesive - NuTab

- KING MEDICAL Cables \& Adapters

- MAVIDON Lemon Skin Prep

- PARKER LAB. Electrode Paste

- RADIANT Infrared Skin Thermometer

- 3M CANADA Micropore-Transpore

- D.O.WEAVER Ten20 • NuPrep

Clavis $^{\mathrm{TM}} \cdot$ Chalgren $\cdot$ Inoject $^{\mathrm{TM}}$

Large stock of Hypodermic Needles

Tel 905-833-3545 Fax 905-833-3543

E-mail: soren@kingmedical.com

Web Site: www.kingmedical.com

King Medical L.td.

145 Kingsworth Road

King City • Ontario L7B 1K1

\section{City life on Tland time coly \\ Neurologist}

Victoria, British Columbia

The Division of Neurology of the Vancouver Island Health Authority is recruiting a full-time Neurologist to practise in Victoria commencing December 2010.

In this position, you will work as an attending physician in the Multiple Sclerosis Clinic and direct this clinic. You will join the Division of Neurology and participate in paid on-call coverage with 9 other members. The Division provides services to a 350,000 direct referral population and a tertiary referral population of 750,000 .

FRCPC Neurology certification, Fellowship training in Multiple Sclerosis and a minimum of one year post-Fellowship clinical experience are required.

Victoria is one of Canada's most livable cities-big city amenities with a small-town feel. Discover Victoria-with unlimited possibilities for your career, family and future!

Please forward your CV and the names of three references to: Brenda Warren

Leader Physician Recruitment Email: physicians@viha.ca or fax: 250.716 .7747

VANCOUVER ISL.AND health athority viha.ca 


\section{Life's better here}

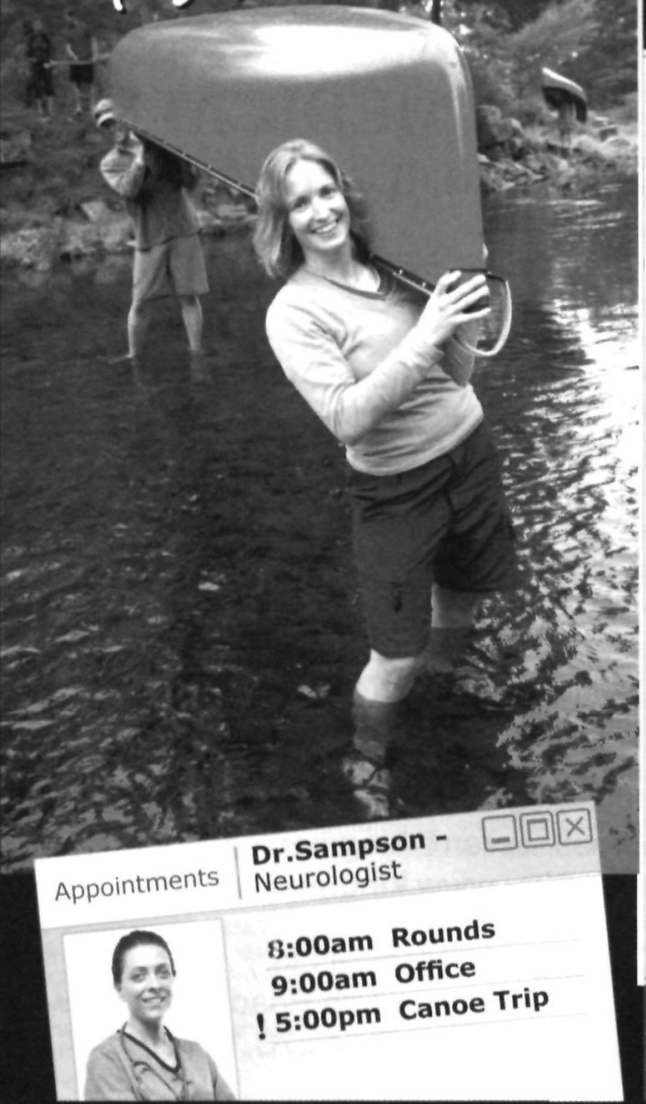

Wanted: A Neurologist Who Believes in Balance Freedom and tranquility at your doorstep.

You've worked hard.

Made sacrifices.

Earned a lifestyle that offers rewarding work and outstanding recreation. The time is now.

Paddle a shimmering lake at daybreak. Catch dinner.

Browse the farmer's market for fresh fixings.

Invite new friends in for an organic feast.

We don't talk about work/life balance. We live it.

We are looking for Neurologists who want to work hard, and play like there's no tomorrow in Kamloops, Kelowna, Penticton and Trail. Physicians in Interior Health communities are eligible for competitive recruitment and retention allowances and generous on-call packages.

What are you waiting for?

Life's better here.Visit betterhere.ca to find out why.

Kamloops Kelowna Penticton Trail

1-877-522-9722 physicianrecruitment@interiorhealth.ca

\section{NOTES}




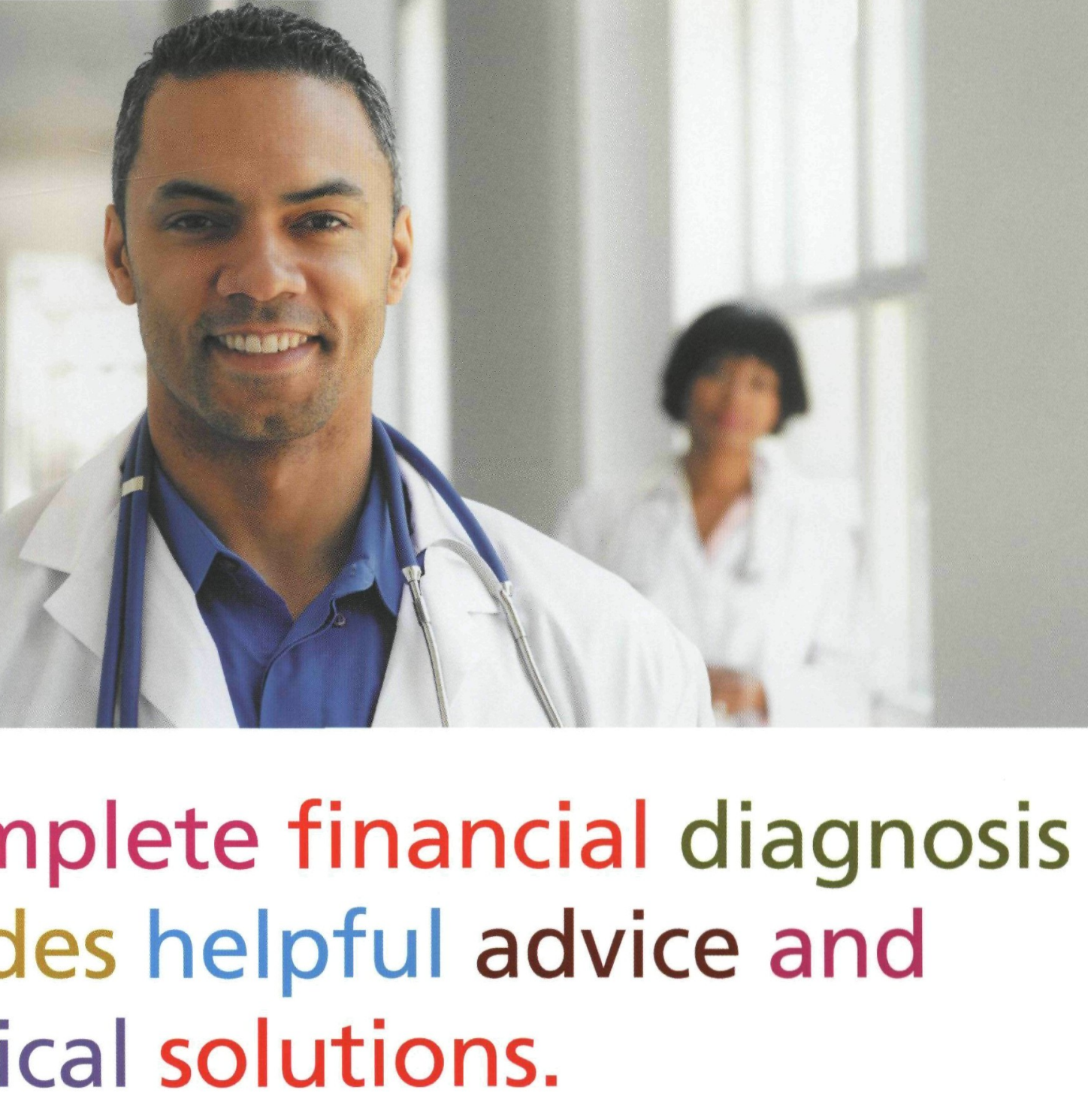

At Scotiabank, we have experts that can help you grow your business. Our Scotia Professional ${ }^{\circledast}$ Plan is a customized financial package that includes everything you need to set up and run a successful practice. You'll get competitive financing rates, flexible payback plans, and a dedicated advisor - all in one convenient package to meet your day to day banking, financing, and investment needs. It makes managing your money easy, so you can focus on serving your patients and growing your practice.

Learn more at any Scotiabank branch or visit www.scotiabank.com/professional

Scotia Professional Plan

You're richer than you think: Scotiabank ${ }^{\circ}$ 


\section{Athena Diagnostics}

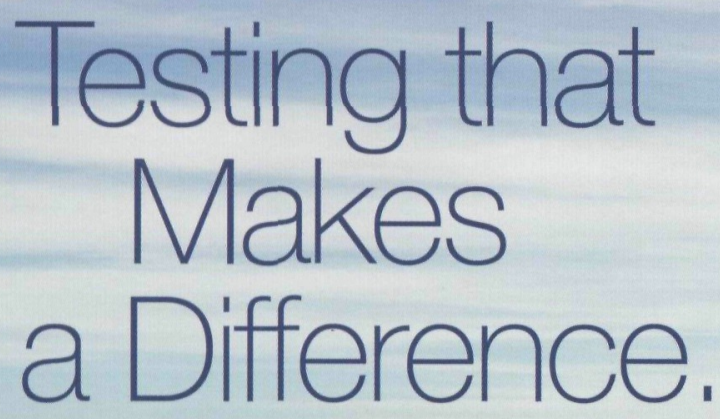

\section{Athena is Your Source for Advanced Neurology Diagnostics}

A Sample of Our Tests Include:

\section{Epilepsy}

- Febrile Seizures Evaluation (\#548) SCN1A, SCN1B, GABRG2

- Complete Tuberous Sclerosis Evaluation (\#556) TSC1, TSC2

\section{Myasthenia Gravis}

- AChR/MuSK Reflexive Antibody Test (\#483) Includes MuSK quantitative titers

\section{Peripheral Neuropathy}

- Complete CMT Evaluation (\#404) 15 genes including PMP22, MFN2 and Cx32

\section{Motor Neuron Disease}

- Complete ALS Evaluation (\#723) SOD1, FUS, TARDBP, ANG, FIG4

See our complete menu at www.AthenaDiagnostics.ca

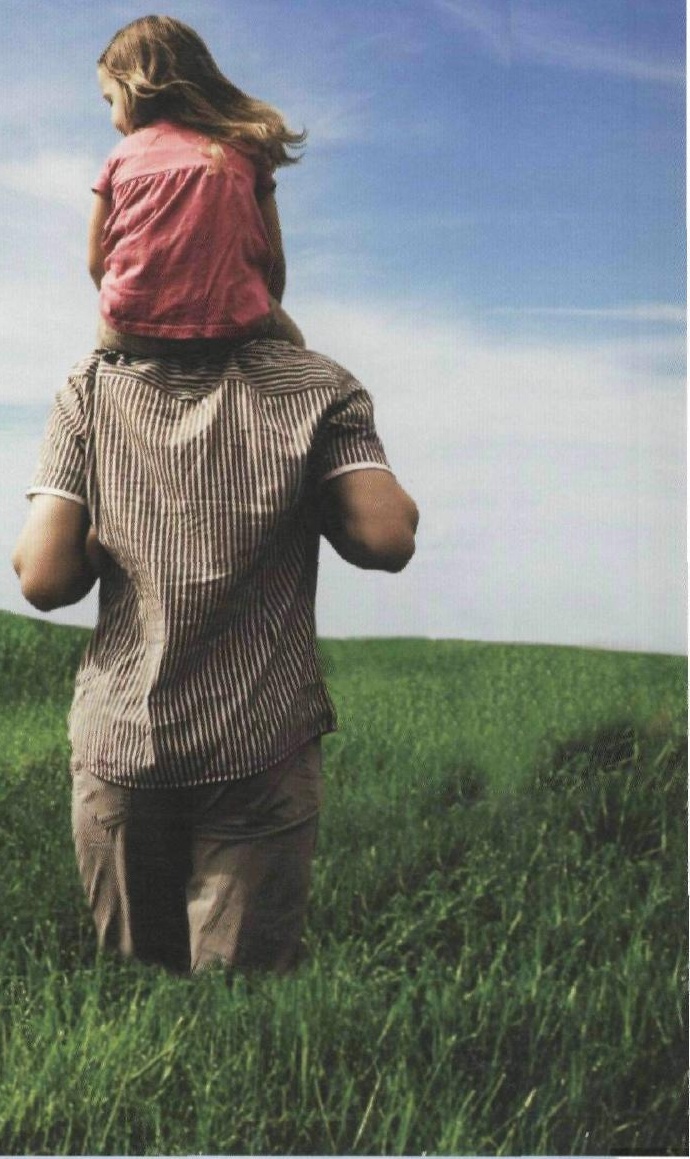

\section{Athena's Tests are EASY to Order!}

- Simple 5-step ordering instructions at www.AthenaDiagnostics.ca

- Athena stores blood samples awaiting Ministry of Health approval so you can order, draw and ship on same visit

- Free FedEx shipping and shipping kits included to reduce send-out costs

For more information, call toll-free (800) 394-4493, Option \#2.

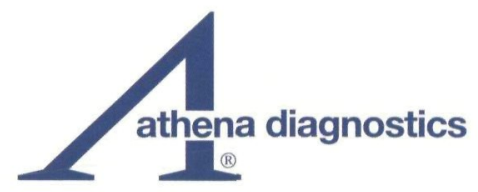

Testing that Makes a Difference. 